\title{
The Key Problem and Solution of Medical Waste High-temperature Steam Treatment
}

\author{
Hongjun Teng \\ School Hospital \\ Tianjin Agricultural University \\ Tianjin, China \\ Hongjun-teng@163.com
}

\author{
Zhenbo BAO \\ Engineering and Technology College \\ Tianjin Agricultural University \\ Tianjin, China \\ baozhenbo@sohu.com
}

\author{
Dengchao JIN \\ Engineering and Technology College \\ Tianjin Agricultural University \\ Tianjin, China \\ jindengchao@163.com \\ Yang LI \\ Engineering and Technology College \\ Tianjin Agricultural University \\ Tianjin, China \\ liyang.tjac@gmail.com
}

\begin{abstract}
Medical waste is directly or indirectly infectious, toxic and other hazardous waste, and high-temperature steam sterilization is a widely used non-incineration technology for centralized treatment medical waste. Basing on the analysis of flow chart of medical waste hightemperature steam treatment, the specific contents of medical waste high-temperature steam treatment technology process, and the graph of high-temperature steam sterilizer pressure or temperature changing with time, the key problem of heat penetration time in high-temperature steam sterilization technology is pointed out, and the problems caused by the undetermined heat penetration time are analyzed. Medical waste is moisture porous media and it has the properties of porous media that interaction interphase, structural diversity, multi-scale, distribution randomness and so on, the research on porous media calorific value heat transfer has made tremendous progress. At last, concluded that conducting research on the heat and mass transfer mechanism of high-temperature steam in medical waste porous media can provide a scientific basis for reasonably determine the heat penetration time.
\end{abstract}

Keywords- Medical Waste; High-Temperature Steam; Sterilization; Non-Incineration Disposal Technology; Porous Media

\section{INTRODUCTION}

Medical waste is directly or indirectly infectious, toxic and other hazardous waste generated in the medical, prevention, health care, teaching, research and other related activities of various medical and health institutions. In the Basel Convention, medical waste is classified in Y1 group to be controlled waste categories, its risk characteristics is grade 6.2. Medical waste is the top waste of 47 categories hazardous waste in China's National List of Hazardous Waste, its number is the first. The random discharge of medical waste without proper treatment can not only damage the water, air and soil of environment, and lead to epidemic infectious diseases and directly endanger people's health $[1,2]$.

\section{HIGH-TEMPERATURE STEAM STERILIZATION IS A WIDELY USED NON-INCINERATION TECHNOLOGY FOR CENTRALIZED TREATMENT MEDICAL WASTE}

The bursting of "SARS" epidemic in 2003 has caused people's great attention on medical waste harm, management and disposal. June 2003, the State Council issued the Medical Waste Management Regulations; October 2003, the Ministry of Health issued Medical and Health Institutions of Medical Waste Management Approach; December 2003, the State Environmental Protection Administration issued Medical Waste Disposal Technical Specifications; January 2004, the State Environmental Protection Administration issued National Hazardous Waste and Medical Waste Disposal Facility Construction Plan. The enactment of above laws, regulations and codes, in the form of law provisions must be centralized disposal of medical wastes.

Medical waste centralized disposal technologies can be divided into two major categories of incineration and non-incineration; different disposal technologies have their respective advantages, disadvantages and applicability [1]. Medical waste high-temperature steam sterilization technology as a non-incineration technology is currently applied more widely around the world. Despite medical waste high-temperature steam treatment also has shortcomings, such as the processing range limitations, the need for strict classification management of medical waste, in the process can produce unpleasant sickening smell, and the reduction degree of volume and quality is not higher. However, medical waste hightemperature steam technology also has the following advantages: the location is relatively easy, no dust and dioxin pollution, small investment, low operation cost and flexible operation, and can be intermittent operation, etc $[1,2]$. August 2006, the State Environmental Protection Administration promulgated and implemented Technical Specifications for Steam-based Centralized Treatment Engineering on Medical Waste (HJ/T276-2006), to guide medical waste high-temperature steam centralized 
treatment project planning, design, construction and operation management.

Medical waste high-temperature steam technology in small and medium-sized medical waste disposal has an irreplaceable advantage, which is suitable for the disposal of medical waste that the production is not much (less than $10 \mathrm{t} / \mathrm{d}$ ), and the yield relatively unstable, and the disposal equipment of medical waste can be intermittent and flexible operation on the basis of how much the amount of medical waste $[2,3]$.

\section{HIGH-TEMPERATURE STEAM STERILIZATION TECHNOLOGY PROCESS AND TECHNICAL PRINCIPLES}

A typical medical waste high-temperature steam treatment process is shown in Fig .1, the process uses intermittent operation mode: first high temperature sterilization, after crushing treatment [3, 4].

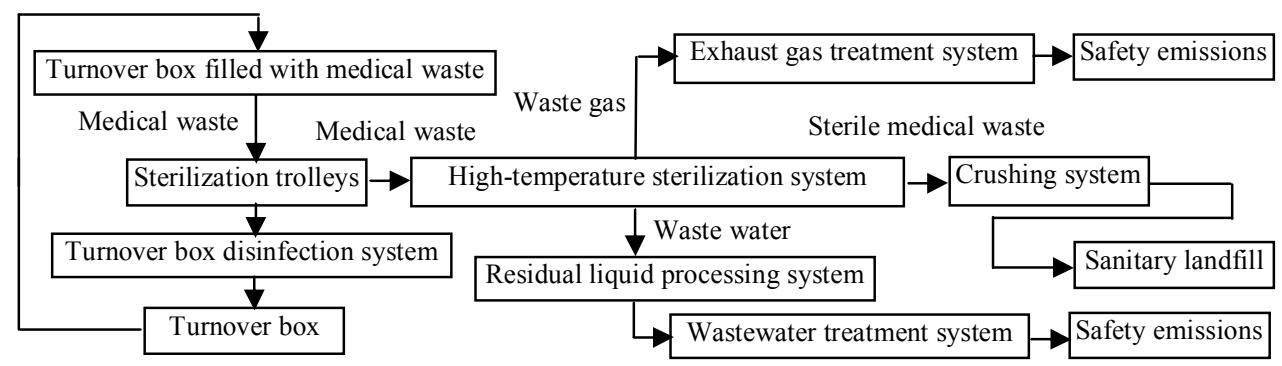

Figure 1. Flow chart of medical waste high-temperature steam treatment time

TABLE I. THE SPECIFIC CONTENTS OF MEDICAL WASTE HIGH-TEMPERATURE STEAM TREATMENT TECHNOLOGY PROCESS [3, 5]

\begin{tabular}{|c|c|c|}
\hline No. & Process & Specific contents \\
\hline 1 & Loading waste & The sterilization trolleys of medical waste are placed into sterilizer and the seal door are closed. \\
\hline 2 & Pre-vacuum & Vacuum pump extracts the air out of sterilizer to reach the set vacuum level $(0.08 \mathrm{Mpa})$ \\
\hline 3 & $\begin{array}{l}\text { Fractionated- } \\
\text { vacuum }\end{array}$ & $\begin{array}{l}\text { The first time into the high temperature saturated steam, the pressure of sterilizer chamber rises abruptly, } \\
\text { reaches the set pressure to stop; and again evacuate and injects the steam, so that the pressure reaches the set } \\
\text { value, repeats above operation three times. After vacuuming the exhaust air is more than } 98 \% \text {, which not only } \\
\text { reduces the content of non-condensable gas in the sterilization, eliminates the interference of air to ensure the } \\
\text { saturated steam can quickly penetrate into the interior of medical waste; and also achieves the purpose of } \\
\text { preheating. The condensate produced in the preheating process is timely discharged out of extravehicular to } \\
\text { increase the efficiency of the steam. }\end{array}$ \\
\hline 4 & Heating up & $\begin{array}{l}\text { After the end of the pulsation vacuum, continuously fills with steam into sterilizer until the sterilization } \\
\text { temperature is raised to the set temperature (not lower than } 134^{\circ} \mathrm{C} \text {, the gauge pressure not less than } 220 \mathrm{kPa} \text { ). }\end{array}$ \\
\hline 5 & $\begin{array}{l}\text { Thermal insulation } \\
\text { sterilization }\end{array}$ & $\begin{array}{l}\text { The sterilization temperature is maintained for } 45 \text { minutes, all micro organisms in medical waste will be all } \\
\text { killed, and the sterilization is completed. }\end{array}$ \\
\hline 6 & Pumping vacuum & Opens again vacuum pumps and vacuums (vacuum degree is generally $0.06-0.09 \mathrm{MPa})$ \\
\hline 7 & Drying & After drying the water content of medical waste is not more than $20 \%$ of the total weight. \\
\hline 8 & Breaking vacuum & $\begin{array}{l}\text { The air inlet valve of sterilization chamber automatically opens to communicate with the outside, until the } \\
\text { pressure of sterilization chamber reaches equilibrium with the external pressure. }\end{array}$ \\
\hline 9 & $\begin{array}{l}\text { Waste shifted out } \\
\text { sterilizer }\end{array}$ & $\begin{array}{l}\text { Opens the sterilization chamber door, the sterilization trolleys of medical waste are launched out sterilization } \\
\text { chamber to crushing system link. }\end{array}$ \\
\hline
\end{tabular}

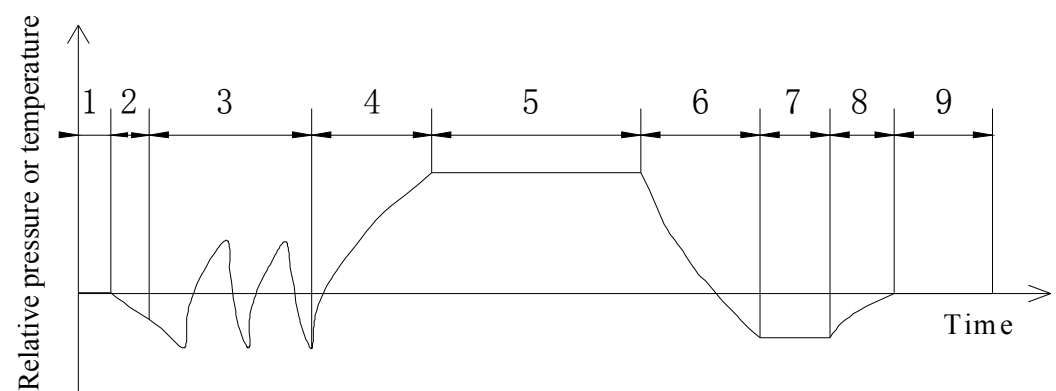

1-loading waste 2-pre-vacuum 3-fractionated-vacuum 4-heating up 5-thermal insulation sterilization 6-pumping vacuum 7-drying 8-breaking vacuum 9-waste shifted out sterilizer

Figure 2. The graph of high-temperature steam sterilizer pressure or temperature changing with time

The principle of medical waste high-temperature steam disposal technology is moist heat sterilization; the sterilization effect is measured by sterilization rate index, with the percentage form to represent the number of microbial death after steam treatment. Since the heat resistance of bacillus stearothermophilus spores in moist heat sterilization is strongest, at home and abroad, the sterilization degree of bacillus stearothermophilus spores is as sterilization level assessment. High-temperature 
steam sterilization rate is not less than $99.99 \%$ in China [1, $2]$.

In different stages of the sterilization process, the sterilizer pressure (temperature) changes graph over time

IV. HEAT PENETRATION TIME IS THE KEY PROBLEM IN HIGH-TEMPERATURE STEAM STERILIZATION TECHNOLOGY

To obtain a high level sterilization effect is the job of high-temperature steam sterilization, so, what are the key factors that influence high-temperature steam sterilization level? According to the law of pressure (temperature) in high-temperature steam sterilizer changing with time, as shown in Fig.2, in spite of high-temperature steam in the pre-vacuum and fractionated-vacuum stage can make the temperature of steam sterilizer rise, has a certain function of sterilization to the medical waste. But due to time is shorter, the temperature is not high, so the sterilization efficiency is not high. The real decisive sterilization is the thermal insulation sterilization phase; the sterilization temperature and time in the phase are the key factors that decide the sterilization effect $[3,5]$.

Sterilization temperature is in the stage of thermal insulation sterilization stage, at the level of moist heat sterilization reaches $99.99 \%$, the stable and effective steam temperature that the sterilizer requires. Sterilization time is in the stage of the thermal insulation sterilization, when the sterilization temperature of sterilization chamber reaches the set value, the continuous residence time of medical waste in the sterilization chamber (not including heating, breaking vacuum and drying time). Sterilization time is started calculating after the internal temperature of medical waste has reached the set sterilization temperature, including thermal death time and heat penetration time [3, $6]$.

Thermal death time refers to the required time that the pathogenic micro organisms reach a certain killing rate at the condition of the set sterilization temperature. Thermal death time mainly depends on the degree of micro organisms heat resistant, because the bacillus stearothermophilus spores strongest heat resistance in the moist heat sterilization, therefore, both at home and abroad, the death time of bacillus stearothermophilus spores is defined as the thermal death time.

Heat penetration time refers to the required heat transfer time, when the heat of sterilization chamber into the inside of medical waste to make all points of medical waste reach the same sterilization temperature and achieve the required level of sterilization.

Sterilization temperature and sterilization time are closely related, the higher sterilization temperature, the shorter thermal death time. Within a certain temperature range, heat penetration time will be shortened with the sterilization temperature increases. However, too high temperature sterilization can bring some negative effects, such as: the sterilization temperature of chamber higher than $138{ }^{\circ} \mathrm{C}$, the bags containing medical waste will be shrinked too much, the leachate and gas of bags are difficult to discharge, resulting in "cold island effect" to affect steam heat transfer efficiency, and heat penetration required time will be extended; the plastic components in medical waste contain PVC more, under too high temperature the plastic will be decomposed and the $\mathrm{HCl}$ gas is quickly released; the higher the temperature setting is shown in Fig .2 [3]. The specific contents of medical waste high-temperature steam treatment technology process are shown in Table 1.

of sterilization, the technical requirements for sterilization equipment will increase, and a corresponding increase in equipment investment costs $[3,5]$. Therefore, in the related technology standards and equipment operation process, the sterilization temperature is set not more than $135^{\circ} \mathrm{C}$.

Under a certain sterilization temperature, the thermal death time is determined, therefore, heat penetration time is a key factor affecting the sterilization effect [6]. How to scientifically determine the heat penetration time? Research scientists at home and abroad have done a lot of work, it can be concluded: the physical characteristics of medical waste, sterilization temperature, the air exclusion circumstances of sterilization chamber, the air in medical waste interstices, and the materials, methods and loading of medical waste packaging all affect the heat penetration time $[2,7]$.

However, due to the following two disadvantages of research work:

- Ignore the essential characteristics that medical waste is the porous medium itself.

- Not from the starting point that heat transfer and mass transfer of high-temperature steam in medical waste porous media, to carry out research on the mechanism of high-temperature steam heat transfer and mass transfer in medical waste porous media, to deeply carry out experimental study and model simulations verify the coupling factors affecting the heat penetration time.

Because the heat and mass transfer mechanism of high-temperature steam in medical waste porous media is not clear, the scientific basis to reasonably determine heat penetration time is lacked.

\section{THE PROBLEMS CAUSED BY THE UNDETERMINED HEAT PENETRATION TIME}

In order to ensure the effect of medical waste hightemperature steam sterilization, at home and abroad have developed relevant treatment technical standards and process, wherein the sterilization temperature and time must be clear. Due to the lack of scientific evidence to determine the heat penetration time, the sterilization time in different standards is greatly vary: for example, EU standards stipulate sterilization temperature $132{ }^{\circ} \mathrm{C}$ and sterilization time 15 minutes; Chinese Technical Specifications for Steam-based Centralized Treatment Engineering on Medical Waste (HJ/T276-2006) stipulate sterilization temperature $134{ }^{\circ} \mathrm{C}$ and sterilization time 45 minutes [4].

The research group had twice revised technical standards Technical Specifications for Steam-based Centralized Treatment Engineering on Medical Waste (HJ/T276-2006), but because lack of scientific basis for the determination of the thermal penetration time, how reasonably determine sterilization time still lack a strong basis.

In addition, the research group for many years engaged in the research and development of medical waste high-temperature steam treatment equipment, in the 
equipment operations found that due to the heat and mass transfer mechanism of high-temperature steam in medical waste porous medium is not clear enough, the impact factors and the law understanding of heat penetration time is not enough clear, serious impacting on the optimal design work of high-temperature steam treatment process. The lack of reasonable and scientific process provisions for the physical characteristics of medical waste, sterilization chamber air exclusion, the air in medical waste interstices, and the materials, methods and loading of medical waste packaging and other technology contents, which not only affects the operating efficiency of disposal equipment, also can lead to medical waste harmless disposal incomplete.

\section{THE SOLUTIONS TO DETERMINE THE THERMAL PENETRATION TIME}

Conducting research on the heat and mass transfer mechanism of high-temperature steam in medical waste porous media can provide a scientific basis for reasonably determine the heat penetration time.

\section{A. The research on porous media calorific value heat transfer has made tremendous progress}

Porous media is a mixed multiphase material having pores or voids in interior, usually constitutes by porous solid skeleton and its single-phase or multi-phase fluid medium within the pores. Solid skeleton pore space communicates with each other; gas phase, liquid phase, or gas-liquid phase flow in the pores. When the liquid and gas share pore space, the porous media is also known porous media [8]. Porous media widespread in nature, industrial and agricultural production and people's daily life, for example, the various minerals, soil; various insulation materials, porous heat pipes and other industrial materials; textiles, paper and other daily necessities all have porous characteristics [9].

The research of heat and mass transfer in porous media has a profound impact on scientific development and technological innovation, and has applied in many fields, such as: transfer process of thermal insulation material; convective drying of food, medicine and agricultural; insulation effect of porous or fibrous material used in construction, etc [10].

\section{B. Medical waste is a porous media}

Medical waste is moisture porous media; it has the properties of porous media that interaction interphase, structural diversity, multi-scale, distribution randomness and so on. The composition of medical waste consist of dense materials (wet weight ratio of $25-70 \%$ ), such as glass, metal, plastic and so on; and porous media material composition (wet weight ratio of $25-60 \%$ ), such as waste paper, gauze and so on $[2,5]$.

For glass, metal, plastic and other dense medical waste, the bacteria build up on the outer surface of medical waste, therefore belong to the surface sterilization process, that is as long as the surface of dense medical waste sterilization temperature reaches the set temperature and keeps sterilization certain time, can achieve the appropriate sterilization effect. For dense material, the main influence factors on heat penetration time are the heat transfer; the forms of heat transfer include heat conduction, convection and radiation, etc. Hightemperature steam can instantly make denseness material surface temperature arise to achieve the temperature requirements of sterilization; the heat penetration time is the desired time that the surface temperature reaches the sterilizing temperature, maintaining a certain time to complete sterilization $[2,5]$.

For the paper, gauze and other porous media materials, pathogenic micro organisms not only build up on the outer surface of these objects, may also exist within the pores of the material; not only the surface reaches the sterilization temperature, but also the internal pores of each point reaches sterilization temperature to ensure the sterilization effect. The thermal penetration time is the required time for the centre of material to reach the sterilization temperature.

The pile form of medical waste also has porous media material properties. The porous media solid skeleton components of medical waste include glass, metal, plastic and other dense materials; and paper, gauze and other porous media materials. There are pores between the solid skeleton of medical waste, in which filled with a small amount of liquid and gaseous. In the high-temperature steam moist heat sterilization process, there are steam and steam condensate in the pores of porous media. Therefore, medical waste also has porous property on a macro scale which also affects the heat and mass transfer processes.

Heat and mass transfer have a complex relationship with sterilization temperature, degree of residual air in sterilizer, the types of medical waste, and pile density of medical waste and other factors $[1,5]$.

\section{The research on high-temperature heat and mass transfer in medical waste porous media can provide a scientific basis for determining the thermal penetration time}

In the high-temperature steam moist heat sterilization process, the steam molecules transfer heat to medical waste, meanwhile in the form of fluid penetrate into the medical waste. Steam instantly entering into closed sterilizer of containing medical waste, due to the role of the temperature difference, some of steam condense to release latent heat of condensation, and to pass into medical waste, in the process there are both thermal conductivity and heat convection. Uncondensed steam molecules at the action of pressure difference and the concentration difference, carry the heat to diffuse to the interior of medical waste. i.e., in the process at the same time there are heat transfer and mass transfer. The liquid in medical waste can evaporate and has bidirectional phase transition with steam [9, 12]. When filled with steam, liquids and other fluids in the air gap of the porous media, the heat in the skeleton and the fluid medium can be transmitted in the form of heat conduction.

Mass transfer process is more complex, in addition to the performance of the fluid flow occurs due to capillary action and diffusion occurs due to concentration difference, but also between fluids of different phases occurs evaporation or condensation $[5,11]$. In addition, accumulate porous media can take place morphological change due to high temperature heat [8].

In summary, medical waste high-temperature steam sterilization is a process of heat and mass transfer in 
medical waste porous media, the heat and mass transfer process is the internal factors to determine the heat penetration time. Therefore, to reasonably determine the heat penetration time, must study the basic theory of hightemperature steam heat and mass transfer in medical waste porous media.

\section{CONCLUSIONS}

From the starting point that medical waste mostly is porous media and its state of accumulation also shows porous; basing on the analysis and experimental research on dimensionality, porosity, and thermal conductivity of medical waste porous media; using the fractal theory and simulation research tools to carry out experimental and modelling studies on high-temperature steam heat and mass transfer in medical waste porous media; and analyzing the influence factors and laws of heat penetration time, can reveal the mechanism of heat and mass transfer, obtain scientific evidence to determine heat penetration time, and provide a theoretical basis for the equipment design and national standards formulation of medical waste high-temperature steam treatment $[8,10]$.

\section{ACKNOWLEDGMENT}

The research work was supported by Tianjin SME Technology Innovation Fund No.13ZXCXSF07000 and Tianjin Science and Technology Special Commissioner Project No. 14JCTPJC00528.

\section{REFERENCES}

[1] Bao Zhen-Bo, Jin Deng-Chao, Teng Hong-Jun and Li Yang, "The General Process of Medical Waste High Temperature Steam Sterilization Treatment Technology", Advanced Materials Research, vol. 807, Sep. 2013, pp. 1160-1163, doi:10.4028/www.scientific.net/AMR.807-809.1160.
[2] Jin Jun-Ping, Gou Dong-Mei, Gao Bo and Qu Wei-Guo, "Engineering Design for High-temperature Steam Treatment Process of Medical Waste", Environmental Sanitation Engineering, vol. 21, Jan. 2013, pp. 32-35.

[3] Gu Liang-Ping, "Study on the High-temperature and High-pressure Steam Sterilization of Medical Waste Treatment System and Application",MS., Hefei University of Technology, China, 2010,pp. 8-30.

[4] The State Environmental Protection Administration, "Technical Specifications for Steam-based Centralized Treatment Engineering on Medical Waste (HJ/T276-2006)", China Environmental Science Press, China, 2006, pp. 5-8.

[5] Wang Shuang-Ping, "Research on the Theory of Heat Mass Transfer in Steam Sterilizing Treatment Process for Medical Waste”, MS., Tianjin University, China, 2007, pp. 5-40.

[6] Pei Zhao-Tang, Bai Xue-Mei and Xiu Fei, "The Process Design of Treating Medical Wastes by High Temperature Steam Sterilization”, Environmental Engineering, vol. 21, Jan. 2009, pp. 58-61.

[7] Bao Ya-Xian, Rao Han-Dong and Li De-Xi, "Design of Process for Treating Medical Wastes with High Temperature Steam", Environmental Engineering, vol. 28, Apr. 2010, pp. 53-55.

[8] Tian Ming-Wei, "Analysis of Heat Transfer of Multi-phase Fibrous Materials under Unsteady-state Conditions", Ph.D., Southeast University, China, 2012, pp. 1-12.

[9] Li Rui-Chuan, "Study on Percolation and Drying of Porous Media by Using Fractal Theory", MS., Kunming University of Science and Technology, China, 2012, pp.1-15.

[10] Li Liu-Ren, Yuan Shi-Yi and Hu Yong-Le, "A New Model for Describing the Relationship between the Permeability and the Porosity of Fractal Porous Media", Journal of Xian Shiyou University (Natural Science Edition), vol. 25, Mar. 2010, pp. 5052.

[11] Jin Deng-Chao, Bao Zhen-Bo and Yu Xin-Ping, "Effects of Vacuum on Sterilizing Rate in Medical Waste Steam Treatment Process", Procedia Environmental Sciences, vol. 25, Nov. 2011, pp. 1407-1411, doi:10.1016/j.proenv.2011.12.211

[12] Jin Deng-Chao, Bao Zhen-Bo, Liu Yu-Le and Teng Hong-Jun, "A Model for Heat and Mass Transfer in Medical Waste Sterilization", Chinese Journal of Environmental Engineering, vol. 5, Nov. 2011, pp.2605-2609. 